\title{
Rare Tracheobronchitis Agent in a Patient with AIDS: Bordetella bronchiseptica
}

\author{
Salih Macin,, ${ }^{1,}$ Fatma Nur Akdogan Kittana, ${ }^{2}$ Ahmet Cagkan Inkaya, ${ }^{3}$ Yakut Akyon, $^{2}$ and Serhat Unal ${ }^{3}$ \\ ${ }^{1}$ Sirnak State Hospital, Microbiology Laboratory, Sirnak, Turkey \\ ${ }^{2}$ Hacettepe University Faculty of Medicine, Department of Medical Microbiology, Ankara, Turkey \\ ${ }^{3}$ Hacettepe University Faculty of Medicine, Department of Infectious Diseases and Clinical Microbiology, Ankara, Turkey \\ "Corresponding author: Salih Macin, MD, Sirnak State Hospital, Microbiology Laboratory, Sirnak, Turkey. Tel: +90-5066904590, Fax: +90-4862161225, E-mail: \\ salihmacin@hotmail.com
}

Received 2016 September 22; Revised 2017 May 08; Accepted 2017 June 24.

\begin{abstract}
Introduction: Bordetella bronchiseptica is an aerobic, Gram-negative pleomorphic coccobacillus. It can infect various mammals including cats, dogs, and pigs. Bordetella bronchiseptica rarely infect humans. Infants, immunosuppressed and HIV infected persons, and patients with comorbidities constitute the risk group for B. bronchiseptica infections. Bordetella bronchiseptica may lead to disseminated infection, cavitary pneumonia, and rarely fatal tracheobronchitis and sepsis.

Case Presentation: A patient who was in follow-up due to acquired immunodeficiency syndrome (AIDS) was admitted to our hospital with persistent dry cough and fever for 4 weeks. The clinical history revealed the presence of classical anti-retroviral resistant HIV infection, and the development of blindness in the right eye because of retinitis. The case was considered as febrile neutropenia; the meropenem therapy was started empirically. Even though we were able to get fever response with empirical therapy, cough remained persistent. Throat culture was inoculated into $5 \%$ sheep blood agar and incubated at $37^{\circ} \mathrm{C}$. Gram-negative coccobacillus was detected in the examination. Then, the colonies were loaded to MALDI-TOF-VITEK MS and the disease factor was determined as B. bronchiseptica. We stopped meropenem therapy on 7 th day and administered clarithromycin $2 \times 500 \mathrm{mg}$ orally for 14 days.

Conclusions: In AIDS patients with chronic cough, B. bronchiseptica should be considered as a pathogen causing opportunistic infection. In this manuscript, we report a case of tracheobronchitis caused by B. bronchiseptica in an AIDS patient.
\end{abstract}

Keywords: Tracheobronchitis, HIV Infection, Febrile Neutropenia, Bordetella Bronchiseptica

\section{Introduction}

Bordetella bronchiseptica is a mandatory aerobic bacterium, which has small irregularly stained Gram-negative pleomorphic coccobacillus form. It appears as commensal in upper airway track of many domestic and wild animals (1). Bordetella bronchiseptica is a zoonotic bacterium and it can cause infection in most of the mammalian species such as cat, dog, horse, rabbit, and pig. In humans, $B$. bronchiseptica infection is not common (2). If a human is infected, it is thought that this infection comes from an infected animal with $B$. bronchiseptica (3). Especially in the young children, in those with underlying disease and in HIV-infected individuals with suppressed immune system, B. bronchiseptica infection has appeared more frequent and severe compared to healthy individuals. It is reported that $B$. bronchiseptica causes disseminated infection, cavitary pneumonia, and rarely fatal tracheobronchitis or septicemia in these patients (4).

\section{Case Presentation}

A patient who was in follow-up due to acquired immunodeficiency syndrome (AIDS) was admitted to our hospital with persistent dry cough and fever for 4 weeks.
The clinical history revealed the presence of classical antiretroviral resistant HIV infection and the development of blindness in the right eye because of the Cytomegalovirus (CMV) retinitis. Six months ago, an investigation was done for a mass in his right maxillary area and he was diagnosed with B-cell lymphoma, and accordingly he got radiotherapy for his treatment. On his physical examination, the body temperature was $38.5^{\circ} \mathrm{C}$, the pulse rate was $98 / \mathrm{min}$, and he had cataract in his right pupil and lipodystrophic changes in the bilateral side. We observed a $1 \mathrm{~cm}$ in diameter fistula tract, which displayed elongation to the right maxillary sinus with a white plate in the orifice in the hard plate. The sound of his breath was rough. The lower extremities had lipodystrophic symptoms.

The case was considered as febrile neutropenia; the meropenem therapy was started empirically. Even though we were able to get fever response with empirical therapy, cough remained persistent. We did not detect any reproduction in blood and urine culture taken prior to treatment. Throat culture was inoculated into 5\% sheep blood agar and incubated at $37^{\circ} \mathrm{C}$. After 24 hours of incubation, microscopic examination was done with Gram stain for colonies. Gram-negative coccobacillus was detected in the examination. Then, the colonies were loaded to MALDI- 
TOF-VITEK MS and the disease factor was determined as $B$. bronchiseptica. We stopped Meropenem therapy on 7th day and administered Clarithromycin $2 \times 500 \mathrm{mg}$ orally for 14 days.

\section{Discussion}

In our case, B. bronchiseptica infection was thought to cause tracheobronchitis and chronic cough. These bacteria can cause respiratory diseases, particularly in domestic and wild animals. For example, it may cause tracheobronchitis called 'Kennel Cough' or shelter cough in dogs; it may be a disease factor for atrophic rhinitis in pigs, pneumonia or otitis media in rabbits. Although the mechanism of human transmission is not fully clarified, it is thought that the transmission occurs through droplet from an animal which carries $B$. bronchiseptica especially from dogs with tracheobronchitis (3). The source of infection is generally individual's domestic animals, which are infected with $B$. bronchiseptica. In our patient, we did not detect any contact with domestic animals.

The number of case reports describing $B$. bronchiseptica infection in patients with AIDS is small $(5,6)$. Dworkin et al. reported that $B$. bronchiseptica infection was identified in 9 patients from 41,000 patients with AIDS registered between 1991 and 1998; they could reproduce bacteria in culture only in two cases (7). The presence of the pathogen was shown in cultures from nasal swabs, sputum, and bronchoscopy. In all these patients, CD4+ lymphocyte count was less than 200. In our case, CD4+ T cell count was $10 / \mathrm{mm}^{3}$, which is very low. Beside B. bronchiseptica, other respiratory tract pathogens can be isolated simultaneously in patients with AIDS (6). Bordetella bronchiseptica related respiratory infection can be observed in patients with AIDS as well as in young children. Ting et al. detected pneumonia associated with $B$. bronchiseptica in a newborn with very low birth weight (1). Moreover, de la Torre et al. detected recurrent respiratory infection associated with $B$. bronchiseptica in a 7-month-old baby with immunodeficiency (8).

Bordetella bronchiseptica can reproduce easily in Bordet-Gengou agar, chocolate agar, and Regan-Lowe agar and its colonies begin to appear after 24 hours of incubation. While B. pertussis cannot grow in blood agar, $B$. bronchiseptica can reproduce. Therefore, when ReganLowe agar is used for inoculation, concurrent inoculation to blood agar should be done. In fact, we could detect $B$. bronchiseptica in our patient's throat culture by using 5\% sheep blood agar. Bordetella bronchiseptica is faster in terms of reproduction compared to other Bordetella species (average growth length is 1 - 2 days). It has oxidase, catalase, and urease, which are effective in its pathogenicity. Bordetella bronchiseptica is the only motile species in its genus. Like some Bordetella species, it cannot produce brown pigmentation in heart infusion agar. Considering evolution, $B$. bronchiseptica is the predecessor of $B$. pertussis and $B$. parapertussis and it is a species which can cause infection in a host other than human (4).

Bordetella bronchiseptica and polyclonal Legionella antibodies are cross reactant. Accordingly, DFA (Direct Fluorescent Antibody) cannot be replaced with culture in the identification of $B$. bronchiseptica because of the observation of false results. The presence of $B$. bronchiseptica can be detected with high sensitivity and specificity using 'Propertussis' and 'Pneumoplex' (B. pertussis, Mycoplasma pneumoniae, Chlamydia pneumoniae, Legionella pneumophila, $L$. micdadei) PCR kit (Cosmo Bio Co. Ltd., Japan) (9). The low sensitivity of the culture in the identification of $B$. bronchiseptica, cross reaction problems in DFA, and cost and requirement of qualified professional of PCR method necessitate the search for a method which will be easy, sensitive, and cost effective in diagnosis. For this purpose, measurement of specific protein profiles of microorganisms by a molecular-based method, MALDI-TOF MS (bioMerieux, Marcy l'Etoile, France) system, has been started to be used. The research literature shows successful detection of $B$. bronchiseptica (10). Indeed, after inoculation of throat samples into 5\% sheep blood agar, Gram staining was done and identified pleomorphic coccobacillus loaded to MALDI-TOF MS system. From the result obtained by the device, B. bronchiseptica was observed in the patient.

For the treatment of $B$. bronchiseptica infection of the immune-suppressed patients, erythromycin, trimethoprim-sulfamethoxazole, and ciprofloxacin are used. Bordetella bronchiseptica species are primarily resistant to rifampin and tetracycline, and also some strains may be resistant to amoxicillin. Treatment of the disease is performed with respect to antibiotic sensitivity of the pathogen. Bordetella bronchiseptica can cause chronic cough pneumonia and tracheobronchitis in the immunosuppressive individuals. It is necessary to identify the pathogen and subsequently determine the antibiotic sensitivity; otherwise the infection of $B$. bronchiseptica could be fatal. Since the culture and DFA methods have low sensitivity and PCR has high sensitivity but high cost, MALDI-TOF-VITEK MS method gives successful results in the identification of $B$. bronchiseptica and can identify the pathogen in the patients with HIV. The patients who underwent macrolide therapy can improve. In AIDS patients with chronic cough, B. bronchiseptica should be considered as a pathogen that can cause opportunistic infection. 


\section{Footnotes}

Authors' Contribution: Salih Macin, Fatma Nur Akdogan Kittana, and Yakut Akyon contributed to the conception and design of the work and drafting the manuscript; Ahmet Cagkan Inkaya and Serhat Unal examined the patient, clinically followed up, developed the original idea, collected data, and drafted the manuscript.

Financial Disclosure: The authors report no conflicts of interest.

Funding/Support: No funding was secured for this study.

\section{References}

1. Ting YJ, Ho PL, Wong KY. Bordetella bronchiseptica Pneumonia in an Extremely-Low-Birth-Weight Neonate. AJP Rep. 2011;1(2):83-6. doi: 10.1055/s-0031-1284223. [PubMed: 23705092].

2. Wernli D, Emonet S, Schrenzel J, Harbarth S. Evaluation of eight cases of confirmed Bordetella bronchiseptica infection and colonization over a 15-year period. Clin Microbiol Infect. 2011;17(2):201-3. doi: 10.111/j.1469-0691.2010.03258.x. [PubMed: 20459438].

3. Galeziok M, Roberts I, Passalacqua JA. Bordetella bronchiseptica pneumonia in a man with acquired immunodeficiency syndrome: a case report. J Med Case Rep. 2009;3:76. doi: 10.1186/1752-1947-3-76. [PubMed: 19946552].

4. Mattoo S, Cherry JD. Molecular pathogenesis, epidemiology, and clinical manifestations of respiratory infections due to Bordetella pertus- sis and other Bordetella subspecies. Clin Microbiol Rev. 2005;18(2):32682. doi: 10.1128/CMR.18.2.326-382.2005. [PubMed: 15831828].

5. de la Fuente J, Albo C, Rodriguez A, Sopena B, Martinez C. Bordetella bronchiseptica pneumonia in a patient with AIDS. Thorax. 1994;49(7):719-20. [PubMed: 8066571].

6. Garcia San Miguel L, Quereda C, Martinez M, Martin-Davila P, Cobo J, Guerrero A. Bordetella bronchiseptica cavitary pneumonia in a patient with AIDS. Eur J Clin Microbiol Infect Dis. 1998;17(9):675-6. doi: 10.1007/BF01708357. [PubMed: 9832276].

7. Dworkin MS, Sullivan PS, Buskin SE, Harrington RD, Olliffe J, MacArthur RD, et al. Bordetella bronchiseptica infection in human immunodeficiency virus-infected patients. Clin Infect Dis. 1999;28(5):1095-9. doi:10.1086/514761. [PubMed: 10452641].

8. de la Torre MJ, de la Fuente CG, de Alegria CR, Del Molino CP, Aguero J, Martinez-Martinez L. Recurrent respiratory infection caused by Bordetella bronchiseptica in an immunocompetent infant. Pediatr Infect Dis J. 2012;31(9):981-3. doi: 10.1097/INF.ob013e31825d2e84. [PubMed: 22572751].

9. Khanna M, Fan J, Pehler-Harrington K, Waters C, Douglass P, Stallock J, et al. The pneumoplex assays, a multiplex PCR-enzyme hybridization assay that allows simultaneous detection of five organisms, Mycoplasma pneumoniae, Chlamydia (Chlamydophila) pneumoniae, Legionella pneumophila, Legionella micdadei, and Bordetella pertussis, and its real-time counterpart. J Clin Microbiol. 2005;43(2):565-71. doi: 10.1128/JCM.43.2.565-571.2005. [PubMed: 15695646].

10. Van den Bossche D, De Bel A, De Smet D, Heylen O, Vekens E, Vandoorslaer K, et al. Prevalence of Bordetella holmesii and Bordetella bronchiseptica in respiratory tract samples from Belgian patients with pertussis-like symptoms by sensitive culture method and mass spectrometry. Acta Clin Belg. 2013;68(5):341-8. doi: 10.2143/ACB.3341. [PubMed: 24579240]. 